Jonathan P. Bird and Lev G. Mourokh

\title{
Localizing and detecting single spins in semiconductor nanostructures
}

ABSTRACT. We review recent experiments in which we have used wavefunction coupling between quantum point contacts as an approach to electrically isolate and detect single spins. The physical basis for our experiments is the idea that a well-defined bound state develops in these nanostructures near pinch-off, where the electron density becomes vanishingly low and manybody interactions are consequently enhanced. By coupling the bound state in one point contact to another that serves as a detector, with the nature of this coupling being provided by the mutual wavefunction overlap between the two nanostructures, a Fano resonance is observed in the detector conductance. In this perspective we discuss how this resonance provides an experimental means to characterize the microscopic properties of the bound state that localizes the single spin.

Nanotechnology Perceptions 5 (2009) 61-70

Nonsubscribers: purchase individual article 